being [Short Warwick-Edinburgh Mental Wellbeing scale] and hope (Adult State Hope Scale) scores which have been validated as accurate in detecting fluctuations in hope were assessed up to 60 days follow-up. We calculated mean NHS cost savings and 95\% central range (CR) from bootstrap analysis.

Results Eleven patients were randomised to IPM and thirteen to OPM. There was no statistically significant difference in all-cause mortality during the index episode (1/11 vs $0 / 13$ ) and up to 60 days follow-up (2/11 vs 2/13) [log rank $\mathrm{p}=0.86]$. (Figure 1). Patients randomised to IPM accrued a median of 47 [IQR 36, 51] vs 59 [41, 60] DAOH in OPM $(\mathrm{p}=0.13) .2$ patients randomised to IPM (vs 6 OPM) were readmitted within 60 days $[p=0.31]$. Hope scores increased more with OPM within 30 days after randomisation but by 60 days, dropped to lower levels compared with the group allocated to IPM (not statistically significant). (Table 1). Inpatients' mental well-being score was higher at baseline but more out-patients had increased total well-being scores by the 60-day follow-up visit $(\mathrm{p}=0.04)$. (Table 2$) .100 \%$ patients in both arms were satisfied according to the 'NHS Family and Friends Test' but interestingly 100\% would choose OPM again whilst only $90 \%$ would choose Inpatient care again. Similarly, $100 \%$ carers were satisfied in the OPM arm whilst $60 \%$ only were satisfied if the patient was randomised to inpatient care. $100 \%$ carers would choose OPM again, vs 60\% IPM carers. OPM was associated with mean cost savings of $£ 2,658$ (95\% CR 460 - 4,857) per patient during the index episode.

Conclusions Patients with acute HF randomised to OPM

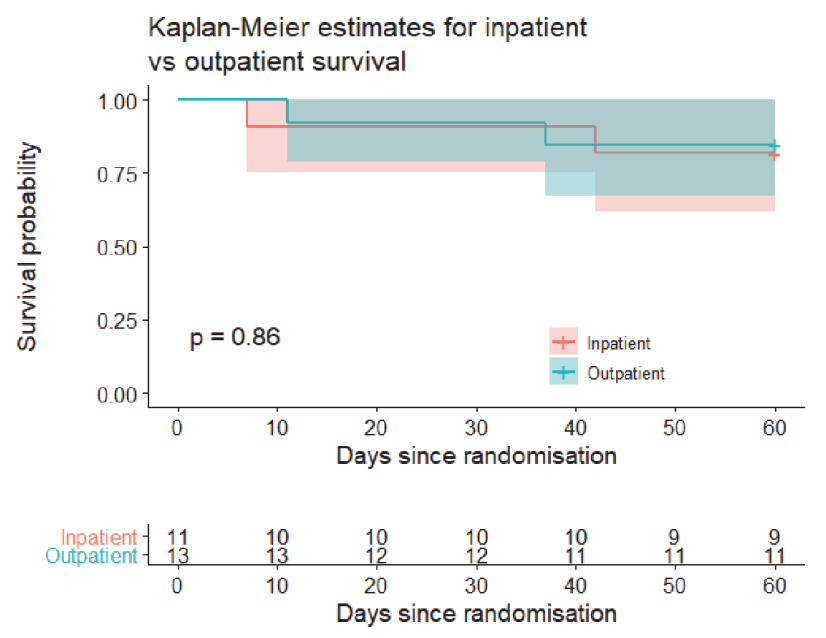

Abstract 152 Figure 1

accrued more DAOH (albeit not statistically significant in this small pilot RCT). There was no increase in mortality but there was a higher risk of readmissions (not statistically significant). OPM was associated with improved mental well-being. But the initial increase in hope diminished within 60 days, possibly as a result of increased readmissions. The pilot RCT generated important hypotheses that need further testing in a large multicentre RCT.

Conflict of Interest none

\section{ANTICOAGULANTS AND ANTIPLATELETS IN COVID-19: IMPACT ON SURVIVAL AND THROMBOEMBOLISM DEVELOPMENT}

Thomas Salmon, Mitchell Titley, Zaid Noori, Mark Crosby, Rajiv Sankaranarayanan. Liverpool University Hospitals NHS Foundation Trust, Liverpool, UK

\subsection{6/heartjnl-2021-BCS.150}

Introduction Higher rates of venous and arterial thromboembolism have been noted in coronavirus disease-2019 (COVID19). There has been limited research on the impact of anticoagulant and antiplatelet choice in COVID-19. We performed a single-centre retrospective cohort study of 933 patients with confirmed COVID-19 infection, investigating the impact of anticoagulation and antiplatelet medication choice on survival and thromboembolism development.

Methods This was a single-centre retrospective cohort study of 933 patients with COVID-19 infection presenting between $01 / 02 / 2020$ and $31 / 05 / 2020$. Survival time at 90 days postdiagnosis and thromboembolism development were the measured outcomes. Patients were grouped by anticoagulant use on first presentation (direct oral anticoagulant, warfarin, therapeutic low molecular weight heparin, and no anticoagulation) and antiplatelet use on admission (aspirin, clopidogrel, dual antiplatelet therapy, or no antiplatelet therapy). Information on pre-existing cardiovascular disease and thromboembolism risk factors was also collected to adjust for confounding variables. Statistical analysis was performed using SPSS version 27.0.1.0.

Results Of 933 total patients, mean age was 68 years and 54.4\% were male. 297 (31.8\%) did not survive at 90 days. A Cox proportional hazards model analysis found no statistically significant relationship between anticoagulant or antiplatelet choice and survival $(\mathrm{p}<0.05) .57(6.3 \%)$ developed thromboembolism. Antiplatelet choice was not shown to have a statistically significant relationship with thromboembolism development. Warfarin and direct oral anticoagulant (DOAC) use did not have a statistically significant impact on thromboembolism development $(\mathrm{p}<0.05)$. Therapeutic low molecular weight heparin (LMWH) use was associated with increased thromboembolism risk (Odds ratio $=14.327$, 95\% CI 1.904 - 107.811, p $=0.010$ ).Additionally, neither ischaemic heart disease, heart failure, or arrhythmia history were found to be associated with decreased survival at 90 days or increased thromboembolism development.

Conclusions Antiplatelet choice was shown to have no impact on survival or thromboembolism development in COVID-19. No patient on dual antiplatelet therapy developed thromboembolism, but this was not found to be statistically significant in multivariate analysis and the sample size was limited $(n=7)$. Anticoagulant choice did not impact survival or thromboembolism development, aside from LMWH. Therapeutic LMWH use was associated with increased risk of thromboembolism. Again, it should be noted that the sample size for patients using therapeutic LMWH was small $(n=4)$, and there may be confounding variables affecting both LMWH use and thromboembolism development. The findings relating to the impact of dual antiplatelet therapy and therapeutic LMWH use on thromboembolism development in COVID-19 should be repeated with a larger sample size and additional adjustment for cofounding variables.

Conflict of Interest The authors have no competing interests to declare 